TENDENCIAS

Revista de la Facultad de Ciencias

Económicas y Administrativas.

Universidad de Nariño

ISSN-E 2539-0554

Vol. XXIII No. 1 - 1er Semestre 2022

Enero - Junio - Páginas 341-371

\title{
ACERCAMIENTO TEÓRICO DE LA RESPONSABILIDAD SOCIAL UNIVERSITARIA (RSU) EN PROGRAMAS DE CONTADURÍA PÚBLICA ${ }^{1}$
}

\section{THEORETICAL APPROACH TO UNIVERSITY SOCIAL RESPONSIBILITY (RSU) IN PUBLIC ACCOUNTING PROGRAMS}

\section{ABORDAGEM TEÓRICA DA RESPONSABILIDADE SOCIAL UNIVERSITÁRIA (RSU) EM PROGRAMAS DE CONTABILIDADE PÚBLICA}

José Luis Villarreal; Carolina Reyes Bastidas; Ángel Javier Mucha Paitan

Magíster en Gestión Empresarial, Universidad Libre, Colombia. Docente investigador Universidad Mariana. ORCiD: 0000-0002-7106-8657. E-mail: jvillareal@umariana.edu.co, Colombia.

Magíster en Administración con énfasis en Negocios, Universidad Autónoma de San Luis Potosí, México. Docente investigador Universidad Mariana. ORCiD: 0000-0002-3903-8597. E-mail: carolreyes@umariana.edu.co, Colombia.

Doctor en Educación, Universidad César Vallejo. Docente investigador, Universidad César Vallejo y Universidad Nacional del Santa. ORCiD: 0000-0003-1411-8096. E-mail: zurdo55@outlook.com.pe, Perú.

Recibido: 2 de junio de 2021

Aprobado: 29 de noviembre de 2021

DOI: https://doi.org/10.22267/rtend.222301.193

\footnotetext{
${ }^{1}$ Artículo de revisión derivado del proyecto de investigación: Percepción de la responsabilidad social universitaria en la formación del contador público de la Universidad César Vallejo (Chimbote- Perú) y Universidad Mariana (PastoColombia) para el año 2020" Financiado por las Universidades Cesar Vallejo (Perú) y Universidad Mariana (Colombia).
} 


\title{
Resumen
}

La Responsabilidad Social Universitaria se establece como estrategia de gestión en instituciones de educación superior, para repensar postulados teóricos, indicadores y criterios. La RSU requiere la participación de los actores de una organización educativa, es transversal a sus actividades y considerando las expectativas de las partes interesadas. La contaduría se ha consolidado como una profesión valiosa para el análisis, aseguramiento y control de información financiera y no financiera importante para la toma de decisiones, con una amplia confianza social fundamentada en lo académico y soportada en el ejercicio profesional. El objetivo del artículo es analizar los fundamentos teóricos sobre la RSU y vincularlo con programas de contaduría pública de la Universidad Mariana (Colombia) y Universidad César Vallejo (Perú). La metodología se ubica dentro del paradigma cualitativo, descriptivo, utilizando la técnica de revisión documental. Como conclusión se identificaron dimensiones, subdimensiones y componentes de la RSU basados en el desarrollo teórico para ser integrados como ejes articuladores en la formación de Contadores Públicos que permita una mejor interacción con el entorno institucional y socio empresarial, de tal forma que su perfil de egreso corresponda a los requerimientos del mundo contemporáneo y se conviertan en actores garantes de transformaciones sociales y cuidado ambiental.

Palabras claves: contabilidad; desarrollo sostenible, educación para el desarrollo sostenible; gestión de la educación; justicia social.

JEL: A22; D63; M41; M14; Q01

\begin{abstract}
University Social Responsibility is established as a management strategy in higher education institutions, to rethink theoretical postulates, indicators and criteria. RSU requires the participation of the actors of an educational organization, it is transversal to its activities and considering the expectations of the interested parties. Accounting has established itself as a valuable profession for the analysis, assurance and control of financial and non-financial information important for decision-making, with broad social trust based on academics and supported by professional practice. The objective of the article is to analyze the theoretical foundations on USR and link it with public accounting programs of the Mariana University (Colombia) and César Vallejo University (Peru). The methodology is located within the qualitative, descriptive paradigm, using
\end{abstract}


the document review technique. As a conclusion, dimensions, sub-dimensions and components of the USR were identified based on the theoretical development to be integrated as articulating axes in the training of Public Accountants that allows a better interaction with the institutional environment and business partner, in such a way that their graduation profile correspond to the requirements of the contemporary world and become guarantors of social transformation and environmental care.

Keywords: accounting, sustainable development; education for sustainable development; educational management; social justice.

JEL: A22; D63; M41; M14; Q01

\section{Resumo}

A Responsabilidade Social Universitária se estabelece como estratégia de gestão nas instituições de ensino superior, para repensar postulados teóricos, indicadores e critérios. A RSU requer a participação dos atores de uma organização educacional, é transversal às suas atividades e atende às expectativas das partes interessadas. A Contabilidade consolidou-se como uma profissão valiosa para a análise, garantia e controle de informações financeiras e não financeiras importantes para a tomada de decisões, com ampla confiança social baseada na academia e amparada na prática profissional. O objetivo do artigo é analisar os fundamentos teóricos sobre a RSU e vinculá-los aos programas de contabilidade pública da Universidade de Mariana (Colômbia) e da Universidade César Vallejo (Peru). A metodologia está inserida no paradigma qualitativo descritivo, utilizando a técnica de revisão documental. Como conclusão, foram identificadas dimensões, subdimensões e componentes da RSU a partir do desenvolvimento teórico a serem integrados como eixos articuladores na formação de Contadores Públicos que possibilitem uma melhor interação com o ambiente institucional e parceiro de negócios, desta forma que seu perfil de graduação corresponda às exigências do mundo contemporâneo e se tornem garantidores da transformação social e do cuidado com o meio ambiente.

Palavras-chave: contabilidade, desenvolvimento sustentável; educação para o desenvolvimento sustentável; gestão educacional; justiça social.

JEL: A22; D63; M41; M14; Q01 


\section{Introducción}

La contaduría se ha consolidado como una profesión valiosa para la preparación, análisis, aseguramiento y control de la información financiera y de interés público, por tanto, juega un papel importante en la toma de decisiones en las empresas e instituciones y cuenta con una amplia confianza social y normativa, su responsabilidad social implica compromisos para el desarrollo sostenible (económicos, sociales y ambientales) que se fundamenta en lo académico y se soporta en el ejercicio profesional (López, 2013). En tanto, según los planteamientos de Brijaldo y Pérez (2017) "la humanidad requiere que se tome conciencia respecto a cómo la acción propia genera implicaciones especificas en su medio. Es posible reconocer cómo desde el actuar correcto del contador público, se podría estar hablando de una contribución al cambio" (p. 4).

El código de ética profesional establece que el ejercicio de la contaduría implica asumir un rol para promover el interés público, que atendiendo a las posturas de Baker citado por Londoño y Bermúdez (2016) motivado en intereses sociales y reconociendo el interés de la sociedad.

Ahora bien, desde lo regulativo, el Congreso de la Republica de Colombia a través de la Ley 43 de 1990, establece que el contador público da fe pública y enfatiza en la función social para el beneficio del orden y la seguridad democrática de las relaciones económicas y sociales. Esta característica, simboliza la confianza de su palabra sobre su trabajo. Por su parte, el congreso de la República del Perú a través de la Ley No. 13253 (1960) actualizada por la Ley 28951 (2007) reglamentó la profesionalización del contador público, resaltando su formación científica y humanista para orientar su práctica profesional a la función social, progreso económico y bienestar de la sociedad; además el año 2005, la junta de decanos del Colegio de Contadores públicos de Perú, publica su Código de ética, con Resolución N 013-2005-JDCCPP. Así las cosas, los profesionales de la contaduría en el contexto colombo peruano, están llamados a desarrollar una gestión contable, financiera, tributaria, aseguramiento y asesoría gerencial de manera estratégica, para tal efecto se requiere de principios y competencias que le permitan crear valor organizacional y con una profunda visión de los impactos socio ambientales de la acción empresarial ante los grupos de interés, esto le permitirá "contribuir positivamente a la sociedad, desarrollar tanto su capacidad crítica como la aptitud práctica para plantear y resolver problemas dentro de un marco ético adecuado, a la vez que se adapta y aprende" (Pulgarin y Zapata, 2014, p. 188). 
Para atender a los requerimientos organizacionales y de la sociedad en función del interés público, se realizan distintos planteamientos para su aplicación práctica en beneficio de la sociedad y bienestar colectivo; uno de estos corresponde a la organización de estandarización contable: Junta Internacional de Normas de Educación Contable (IAESB) (2015), organización independiente dentro del redil de la Federación Internacional de Contadores (IFAC), a través de su comité de educación, en el año 2015 publicó estándares internacionales de educación para el desarrollo de competencias y el objetivo es "ayudar a las organizaciones profesionales de contabilidad, al igual que las organizaciones educativas, los empleadores, reguladores, autoridades gubernamentales y cualquier otra parte interesada que apoye el aprendizaje y el desarrollo de contadores profesionales."

Se destaca que dicho ejercicio profesional, en el marco de las actividades, funciones y competencias están incluidos los principios profesionales, la ética y conocimientos para un mejor desenvolvimiento en el desarrollo de sus labores cotidianas de tipo intelectual, estratégica, normativa - técnica y personal, que permitan fortalecer su aptitud y actitud profesional, aspectos reglamentados en el articulado de la Ley 43 de 1990 en Colombia y en el Perú, con la ley 13253 y su actualización con la norma No. 28951.

De manera particular, en los programas académicos (Universidad Mariana y Universidad Cesar Vallejo) se requiere indagar y generar una reflexión teórica respecto al interés por incorporar en su gestión, planes, procesos administrativos e informes de la RSU vinculados con el desarrollo de las funciones esenciales.

De lo planteado, surge la importancia de insistir en la formación integral del futuro profesional contable, que este mejor preparado para interactuar ante un futuro cambiante, complejo e impredecible (Foster, 2002), donde no solo se dé primacía a la acumulación de temáticas y/o contenidos que pueden quedar obsoletos en un corto tiempo; es necesario desarrollar en los profesionales contables de Perú y Colombia el interés por el cuidado de los recursos naturales (agua, fauna y flora), el fomento por la cultura y promoción de la identidad, la participación ciudadana para fortalecer la democracia y la intervención en proyectos que promuevan la sustentabilidad y justicia social. De acuerdo con Quintero (2018), la contaduría como agente de 
información, control, aseguramiento, certificación y fiscalización debe ser garante de responsabilidad social informando acerca de su cumplimiento y vigilando su inclusión en las prácticas cotidianas de las empresas contemporáneas.

Entonces, las instituciones de Educación Superior (IES) deben abordar dentro del currículo, junto a la formación disciplinar procesos de experiencia vivencial, interacción de conocimientos y análisis de impactos de la toma de decisiones con los diferentes grupos de interés, como el progreso del talento humano, vinculación con partes relacionadas: proveedores de capital y de bienes y servicios, impacto en los usuarios. Unido al fortalecimiento de la identidad y cultura organizacional para la apropiada gestión, la transparencia en el manejo de recursos, la generación y análisis de informes financieros y no financieros para una toma de decisiones inteligentes, innovadoras e incluyentes que promuevan el desarrollo sostenible (Escorcia et al., 2007).

En consecuencia, es menester conocer los retos a los que se expone un profesional contable de carácter financiero, personal y por su puesto de carácter social donde la fe pública del contador, es la que ayuda y permite que la sociedad deposite su confianza en la información que ellos suministran. Así, la RS no solo implica un compromiso con el estado, sino que a su vez es un compromiso personal y desde luego un compromiso académico; donde se debe generar conciencia de la importancia del buen uso de las prácticas contables (Navarro y Saavedra, 2016).

Se debe reconocer, que es compromiso del profesional contable estar al servicio del interés público, su función está centrada en la protección del interés económico y vinculase con temas socioambientales, para avanzar en la agenda 2030 de naciones unidas y gestionar el cambio para un desarrollo sostenible. Así en su desempeño profesional y de acompañamiento a las diversas entidades (públicas y/o privadas), al desarrollar su objeto social y misional no podrán moralmente estar por encima o en contraposición del bien común de la sociedad y los limitados recursos naturales Leff (el medio ambiente). En tanto la educación toma rumbo hacia la sustentabilidad, identificando y legitimando nuevos derechos ambientales, culturales y colectivos, para el cuidado patrimonial y preservación de sus arraigos e identidad cultural (Leff, 2013). 
Unido a lo anterior, el Contador Público en formación será garante y protector de la riqueza en sus dimensiones económicas, sociales y ambientales, para que las próximas generaciones puedan disfrutar de un mejor ambiente, un planeta sostenible y de mejores prácticas de convivencia entre el ser humano con la naturaleza, los demás seres vivos y entre sus semejantes.

Se debe reconocer que las instituciones de educación superior de todo el mundo están desarrollando e implementando la Responsabilidad Social Universitaria (RSU) como parte del proceso de construcción de una estrategia que vincule la RS con los esfuerzos para alcanzar adecuadamente sus tres misiones: docencia, investigación y vinculación con la sociedad. La implementación de prácticas de RSU promueve la calidad de los sistemas de educación superior, así como también impactar positivamente el entorno que los rodea (Teran y Torres, 2020).

Por lo anterior, es necesario que los futuros Contadores Públicos sean formados en instituciones de educación superior que asuman la RSU como un eje transversal en sus funciones sustantivas y su quehacer como universidad, de tal forma que su perfil de egreso corresponda a los requerimientos del mundo contemporáneo y se conviertan en actores garantes de cambio dentro de las organizaciones del sector público, privado y/o de la sociedad en general (Zúñiga et al., 2019). De igual forma Agudelo y Viloria, reflexionan sobre el proceso educativo y manifiestan que a formación requiere de una "intervención" a la interioridad del sujeto contador público, para desarrollar un juicio profesional que permita las decisiones más adecuadas. De lo contrario, las consecuencias de continuar normando conductas sin reconocer al sujeto contador pueden conducir a un conflicto en el profesional entre cumplir una norma que puede ser aceptable desde el código profesional o actuar en razón a sus valores (Agudelo y Viloria, 2017).

Para abordar el objetivo del artículo, después de la introducción se tiene une revisión de la literatura, luego se plantea la metodología, como base para alcanzar los resultados de revisión documental y se finaliza con las conclusiones. 


\section{Revisión de la literatura sobre RSU}

La responsabilidad social tiene sus orígenes en el contexto empresarial, apareciendo por primera vez en 1953, cuando Bowen (1953) la definió como un comportamiento ético de la empresa ante la sociedad y las partes interesadas, y reconoció el espíritu del entorno legal y normativo en el ámbito empresarial. Por su parte, García y Zabala (2008; p.15), se refieren a la RSE como "un instrumento que implica un compromiso de las empresas a través de la aplicación sistemática de recursos para respetar y promover los derechos de las personas, el crecimiento de la sociedad y el cuidado del medio ambiente". Resulta claro que la RSE constituye una herramienta estratégica para dar respuesta a demandas originadas en las dimensiones social y ambiental para alcanzar el objetivo de la rentabilidad de la empresa (Amato et al., 2016; Gasca, 2011). Sin embargo, esta concepción de RSE se encuentra vinculada a una mirada economicista y no como un deber ser que permita generar transformaciones; esto le da a la RSU un carácter aparente y no misional o ético.

En este orden de ideas, Muhammad et al, (2021) afirman que es imperativo que las universidades integren iniciativas de responsabilidad social en sus políticas administrativas y procedimientos de gestión para lograr un impacto significativo. La participación de los stakeholders es necesaria para lograr un enfoque a largo plazo y una transformación significativa de los problemas sociales. Las universidades de todo el mundo deben asumir la responsabilidad social como un proceso integral de la organización y moldear sus actividades de enseñanza, educación y formación en consecuencia. Conjuntamente, afirma que la RSU difiere en naturaleza de la RSE, ya que la naturaleza de las operaciones y los objetivos institucionales son inherentemente diferentes a las de las organizaciones empresariales.

En el ámbito educativo, este concepto encuentra su referente en el funcionamiento de la universidad, debido a que las instituciones de educación superior se vinculan con la comunidad y los intereses de los distintos grupos de referencia académico, empresarial, gubernamental, ambiental y en general la sociedad civil (Gaete, 2011).

Conjuntamente, la Organización de las Naciones Unidas para la Educación, la Ciencia y la Cultura (UNESCO) ha venido planteando, desde el año 1998, que las instituciones de educación superior (IES) están llamadas a generar un mayor compromiso e interactuar con y para los diferentes grupos 
de interés, en el contexto de la sociedad de la información y conocimiento, teniendo en cuenta que son entes especializados en la generación y transmisión del conocimiento científico, lo que se ha denominado como Responsabilidad Social Universitaria (RSU) (UNESCO, 1998).

Avanzando en los fundamentos de la Responsabilidad Social Universitaria RSU, se recurre a invetigaciones que analizan el aporte de éste enfoque de gestión universitaria para integrarse con los grupos de interés, los impactos de su accionar y el desarrollo local, entre estos aspectos se encuentra la necesidad de estudiar la preservación y valoración de la identidad, a partir de una educación para la interculturalidad, con el análisis y comprensión del contexto (capital éndogeno) junto a los retos educativos y sociales de las últimas décadas; en definitiva, la construcción de nuevos saberes y métodos para trabajar con los grupos de interés que permitan priorizar necesidades y fomentar sinergias para avanzar sin dejar a nadie atrás, formando ciudadanos responsables, que rechacen toda forma de violencia, violación de los derechos humanos, discriminación, corrupción, injusticia y concentración de la riqueza.

De igual forma, un verdadero significado de la RSU, requiere que esta sea comprendida en el seno de la comunidad universitaria, analizada como un proceso global dentro de las instituciones universitarias cuya cobertura trasciende las funciones sustantivas logrando el compromiso de toda comunidad universitaria hacia la vinculación social con las comunidades y stakeholders integrados por personas y organizaciones que son influenciados por las IES.

Siguiendo a los autores Aristimuño y Rodríguez (2014), en la Tabla 1 se evidencian algunas de las principales definiciones sobre RSU, las cuales fueron clasificadas desde un enfoque organizacional (tradicional) y/o de transformación (enfoque crítico). 


\section{Tabla 1}

Definiciones de RSU

\begin{tabular}{|c|c|c|}
\hline Autor & Definición & Enfoque \\
\hline $\begin{array}{l}\text { Valleys (2006) } \\
\text { Universidad } \\
\text { Católica de } \\
\text { Lima }\end{array}$ & $\begin{array}{l}\text { "Una política de calidad ética del desempeño de la } \\
\text { comunidad universitaria (estudiantes, docentes y personal } \\
\text { administrativo) a través de la gestión responsable de los } \\
\text { impactos: educativos, cognitivos, laborales y ambientales } \\
\text { que la universidad genera, en un diálogo participativo, con } \\
\text { la sociedad para promover el Desarrollo Humano } \\
\text { Sostenible". }\end{array}$ & Transformación \\
\hline $\begin{array}{l}\text { Universidad } \\
\text { Construye País } \\
\text { (2006) }\end{array}$ & $\begin{array}{l}\text { "Una Universidad es socialmente responsable por la } \\
\text { capacidad prospectiva y transformadora que tiene como } \\
\text { organización social, al difundir y poner en práctica un } \\
\text { conjunto de principios y valores mediante una gestión } \\
\text { inteligente de los impactos: organizacional; ambiental; } \\
\text { educativo; cognitivo y social, producidos por medio de sus } \\
\text { procesos claves: gestión, docencia, investigación y } \\
\text { extensión, a través de políticas éticas de calidad, en diálogo } \\
\text { participativo con la sociedad con quien busca en conjunto, } \\
\text { un desarrollo humano y sustentable y el cual concreta la } \\
\text { demanda ética de justicia y equidad social." }\end{array}$ & Organizacional \\
\hline $\begin{array}{l}\text { La Asociación } \\
\text { de } \\
\text { Universidades } \\
\text { Jesuitas de } \\
\text { América } \\
\text { Latina } \\
\text { (AUSJAL, } \\
\text { 2007) }\end{array}$ & $\begin{array}{l}\text { "Es la habilidad y efectividad de una universidad para } \\
\text { responder a las necesidades de transformación de la } \\
\text { sociedad donde está inmersa, mediante el ejercicio de sus } \\
\text { funciones sustantivas: docencia, investigación, extensión y } \\
\text { gestión". Estas funciones deben estar animadas por la } \\
\text { búsqueda de la promoción de la justicia, solidaridad y la } \\
\text { equidad social, mediante la construcción de respuestas } \\
\text { exitosas para atender los retos que implica promover el } \\
\text { Desarrollo Humano Sostenible." }\end{array}$ & Transformación \\
\hline $\begin{array}{l}\text { Asociación de } \\
\text { Universidades } \\
\text { de Colombia } \\
\text { (ASCUN, } \\
\text { 2007) }\end{array}$ & $\begin{array}{l}\text { "Es el ámbito de encuentro e interacción de saberes de las } \\
\text { comunidades educativas con la sociedad en forma } \\
\text { pertinente, ética, responsable y continúa guardando la } \\
\text { identidad institucional y enriqueciendo el quehacer } \\
\text { académico para aportar a la construcción de país en el } \\
\text { contexto global". }\end{array}$ & Organizacional \\
\hline $\begin{array}{l}\text { Medina, } \\
\text { Franco, Torres, } \\
\text { Velázquez y } \\
\text { Valencia, 2017 }\end{array}$ & $\begin{array}{l}\text { "La categoría responsabilidad es entendida como la } \\
\text { asunción de las obligaciones sociales en un compromiso } \\
\text { consecuente en su actuación, así como mostrar sentido del } \\
\text { deber, la obligación y el compromiso del cumplimiento de }\end{array}$ & Organizacional \\
\hline
\end{tabular}


las principales actividades, tanto en el plano individual como social".

Fuente: elaboración propia con datos de Aristimuño y Rodriguez (2014; p.377) y Medina et al., (2017).

De acuerdo con Vallaeys (2014) la universidad socialmente responsable se vincula con impactos de su organización administrativa, de la formación impartida, de los conocimientos generados por desde la investigación y los impactos que surgen de la interacción con el entorno social y relaciones de extensión (ver Figura 1).

\section{Figura 1}

Impactos de la RSU

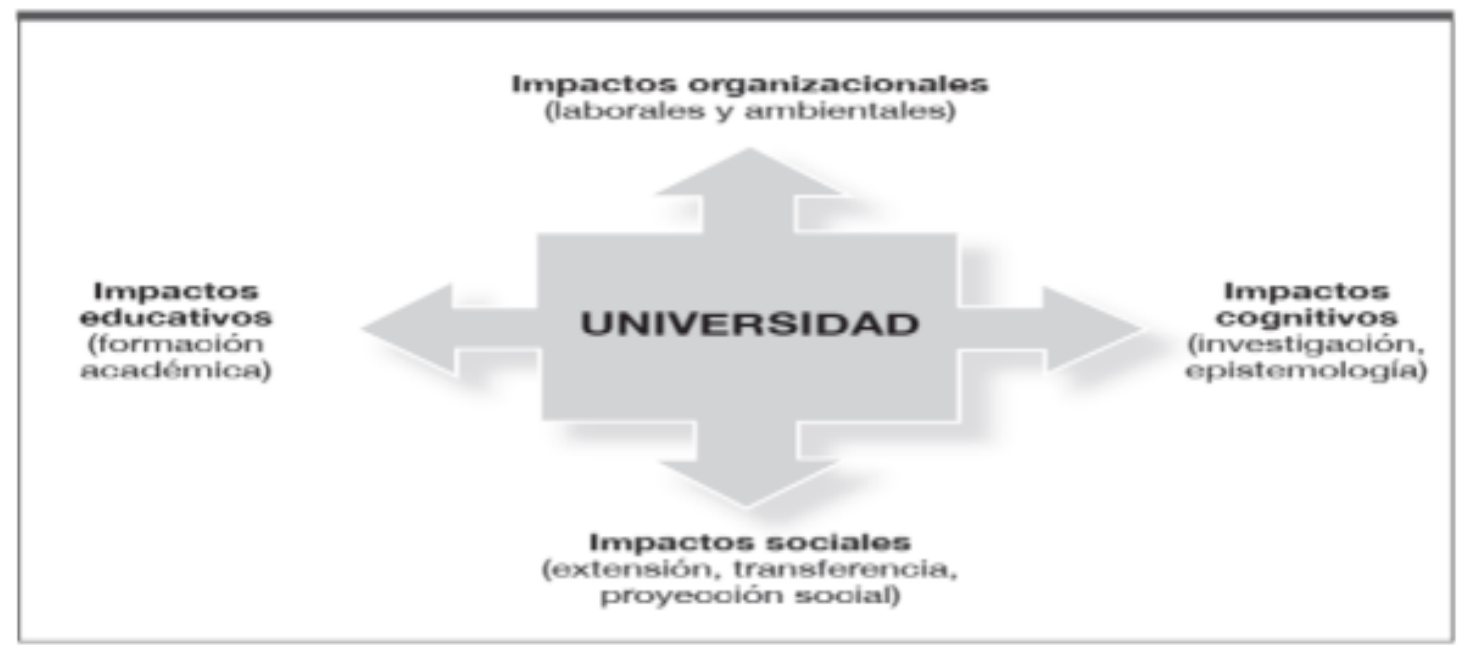

Fuente: Vallaeys, De la Cruz, \& Sasia (2009).

Estos impactos serán la base para el estudio de la percepción por parte de la comunidad universitaria representada por estudiantes, docentes y directivos frente a la RSU y su aporte en la formación del profesional contable.

Específicamente en el contexto Latinoamericano, los cambios económicos, políticos y sociales que tuvieron lugar en las últimas décadas también han tenido un impacto en las instituciones de educación superior, lo que ha generado un amplio proceso de reforma destinado a enfrentar los nuevos desafíos que atañen el mundo de hoy, la globalización, la sociedad del conocimiento, la innovación, el desarrollo de tecnologías y un énfasis creciente en las fuerzas del mercado se 
encuentran entre los factores clave que influyen en la misión, organización, perfil de las universidades y el modo de operación de la educación superior (Vasilescu et al., 2010).

A lo anterior se unen los planteamientos De Sousa (2010), que propone una reforma "creativa, democrática y emancipadora de la Universidad en todo el mundo" (p. 37). Asimismo, Sousa sostiene que el único modo eficaz y emancipador de enfrentar la globalización neoliberal es contraponerle una globalización alternativa, que él llama "globalización contrahegemónica, pero que Wolff (2009) ha denominado Globalización democrática.

Dadas las dinámicas del mundo actual, se debe considerar la inclusión de tecnologías que trae consigo la globalización que, si bien, han generado nuevas formas de organización del trabajo, ha eliminado fuentes de empleo para lo cual se requiere repensar y flexibilizar los planes de estudios en la formación profesional, considerando qué conocimientos y competencias son esenciales en la formación de los universitarios para potencializar su función transformadora de la sociedad.

Así, se debe entender la educación en su carácter formal pero también como dimensión de la vida humana que no solo sirve para el trabajo, sino que aporta beneficios en todas las esferas del quehacer humano; por tanto, la universidad es un insumo que demanda la comunidad, con ciudadanos modernos con sentido socioambiental (Piña, 2006).

No obstante, la masificación de la educación superior en Latinoamérica afronta una comercialización del mundo universitario, con efectos muy cuestionados en la educación profesional y formativa; también los procesos educativos adelantan estrategias de calidad sometida a guías y estándares de acreditación, donde se viven entornos sociales y económicos diferentes. Las funciones sustantivas de extensión en el contexto latinoamericano, son un modelo de participación social de las IES, con el fin de aportar a los retos y desafíos de la globalización (Lanz, 2003; Vallaeys y Carrizo, 2006).

En consecuencia, si la universidad se transforma en institución funcional, únicamente, para responder al capitalismo, excluye los principios de humanización; formación política, democracia, arte, con enfoque planetario sostenible. La apuesta debe estar enfocada a la consolidación de una 
universidad alternativa que asuma el reto de construir, interpretar o develar verdades tanto de la ciencia como de contexto cultural y ancestral, que aporte al desarrollo tecno-científico y económico en condiciones de equidad y bienestar, deseo de una sociedad plural, diversa, justa y con sentido de responsabilidad social (Vélez y Ruiz, 2019).

De acuerdo con Vallaeys y Álvarez (2019), para que exista una transformación real, la RSU tiene que traspasar por todos los actores internos de la universidad, debido a que es transversal a todas las actividades.

Sin embargo, el análisis y la investigación de la responsabilidad social dentro de las universidades es significativamente menor en comparación con el entorno de las empresas, lo que de acuerdo con Hill (2004) se debe a que los académicos de las universidades que investigan estos temas, cuando ocasionalmente dirigen su atención hacia el interior de sus propias universidades.

Conjuntamente, se requiere un concepto específico de RSU que considere las características genuinas de las IES, entidades dedicadas a la construcción y transferencia de saberes humanos y científicos, y que no puede ser reducida a una mera empresa de «servicios educativos» para «clientes» estudiantes (Vallaeys y Álvarez, 2019).

Por tanto, sin prescindir los aportes de la RSE, durante la primera década del presente siglo, las universidades latinoamericanas empezaron un trabajo de redefinición y apropiación de la RSU, en Tabla 2 se pueden evidenciar algunos modelos implementados por universidades Latinas. 


\section{Tabla 2}

Modelos de RSU en Latinoamérica

\begin{tabular}{|c|c|c|c|c|c|}
\hline País & Universidad & RSU & Componentes & Dimensiones & Stakeholders \\
\hline $\begin{array}{l}\text { México } \\
\text { (Universidad } \\
\text { Autónoma } \\
\text { de Yucatan, } \\
\text { 2014) }\end{array}$ & $\begin{array}{l}\text { Universidad } \\
\text { Autónoma } \\
\text { de Yucatán }\end{array}$ & $\begin{array}{l}\text { Conjuntar de } \\
\text { manera } \\
\text { estratégica las } \\
\text { acciones de RSU } \\
\text { que impacten } \\
\text { significativamente } \\
\text { en el contexto } \\
\text { social, económico } \\
\text { y ambiental de la } \\
\text { región y del país }\end{array}$ & $\begin{array}{l}\text { - Ámbito social } \\
\text { - Ámbito económico } \\
\text { - Ámbito empresarial }\end{array}$ & $\begin{array}{l}\text { - Formación } \\
\text { profesional y } \\
\text { ciudadana } \\
\text { - Gestión social } \\
\text { del } \\
\text { conocimiento } \\
\text { - } \text { Gestión } \\
\text { responsable } \\
\text { - Participación } \\
\text { social }\end{array}$ & $\begin{array}{l}\text { Internos } \\
\text { Alumnos, personal académico, } \\
\text { personal administrativo, } \\
\text { directivos, consejeros y sindicatos } \\
\text { Externos } \\
\text { Padres de familia, proveedores, } \\
\text { gobierno, organizaciones sociales, } \\
\text { asociaciones civiles, sociedad en } \\
\text { general, empresas, centros de } \\
\text { investigación y organismos } \\
\text { nacionales e internacionales }\end{array}$ \\
\hline $\begin{array}{l}\text { Ecuador } \\
\text { (Calle \& } \\
\text { Santacruz, } \\
\text { 2011) }\end{array}$ & $\begin{array}{l}\text { Universidad } \\
\text { Politécnica } \\
\text { Salesiana }\end{array}$ & & $\begin{array}{l}\text { - Aspectos: laborales } \\
\text { y ambientales } \\
\text { - Aspectos: proceso } \\
\text { de enseñanza- } \\
\text { aprendizaje y } \\
\text { construcción } \\
\text { curricular } \\
\text { - Aspectos: } \\
\text { Orientaciones } \\
\text { epistemológicas, } \\
\text { enfoques teóricos, } \\
\text { líneas de } \\
\text { investigación y } \\
\text { procesos de } \\
\text { producción y } \\
\text { difusión del saber } \\
\text { - Aspectos: vínculos } \\
\text { de la universidad } \\
\text { con actores }\end{array}$ & $\begin{array}{l}\text { - Impacto } \\
\text { organizacional: } \\
\text { función gestión } \\
\text { - Impacto } \\
\text { educativo: } \\
\text { función } \\
\text { docencia } \\
\text { - Impacto: } \\
\text { cognitivo: } \\
\text { función } \\
\text { investigación } \\
\text { - Impacto social: } \\
\text { función } \\
\text { distinción }\end{array}$ & $\begin{array}{l}\text { Internos } \\
\text { Alumnos, personal } \\
\text { docente/investigador, personal no } \\
\text { docente. } \\
\text { Externos } \\
\text { Egresados, empleadores, } \\
\text { competidores, competidores } \\
\text { locales, estado, }\end{array}$ \\
\hline
\end{tabular}




\begin{tabular}{|c|c|c|c|c|c|}
\hline & & & $\begin{array}{l}\text { externos, } \\
\text { participación en el } \\
\text { desarrollo de su } \\
\text { capacidad social }\end{array}$ & & \\
\hline $\begin{array}{l}\text { Colombia } \\
\text { (Pontificia } \\
\text { Universidad } \\
\text { Javeriana, } \\
\text { 2014) }\end{array}$ & $\begin{array}{l}\text { Universidad } \\
\text { Javeriana- } \\
\text { Cali }\end{array}$ & $\begin{array}{l}\text { “...la habilidad y } \\
\text { efectividad de la } \\
\text { universidad para } \\
\text { responder a las } \\
\text { necesidades de } \\
\text { transformación de } \\
\text { la sociedad donde } \\
\text { está inmersa, } \\
\text { mediante el } \\
\text { ejercicio de sus } \\
\text { funciones } \\
\text { sustantivas: } \\
\text { docencia, } \\
\text { investigación, } \\
\text { extensión y } \\
\text { gestión interna. } \\
\text { Estas funciones } \\
\text { deben estar } \\
\text { animadas por la } \\
\text { búsqueda de la } \\
\text { promoción de la } \\
\text { justicia, la } \\
\text { solidaridad y la } \\
\text { equidad social, } \\
\text { mediante la } \\
\text { construcción de } \\
\text { respuestas } \\
\text { exitosas para } \\
\text { atender los retos }\end{array}$ & $\begin{array}{l}\text { - Aspectos: } \\
\text { integración de la } \\
\text { RSU en el currículo, } \\
\text { experiencia } \\
\text { vivencial, reflexión } \\
\text { y análisis crítico y } \\
\text { perfil del egresado } \\
\text { - Aspectos: } \\
\text { metodología } \\
\text { adecuada a } \\
\text { principios éticos, } \\
\text { interacción de } \\
\text { conocimientos, } \\
\text { socialización e } \\
\text { incidencia. } \\
\text { - Aspectos: clima } \\
\text { organizacional, } \\
\text { desarrollo de talento } \\
\text { humano, relación } \\
\text { con los proveedores, } \\
\text { inclusión, } \\
\text { comunicación } \\
\text { responsable, } \\
\text { participación y } \\
\text { cultura de la } \\
\text { transparencia y de } \\
\text { mejora continua } \\
\text { - Aspectos: gestión de } \\
\text { recursos }\end{array}$ & $\begin{array}{l}\text { - Impacto } \\
\text { educativo } \\
\text { - Impacto } \\
\text { cognitivo y } \\
\text { epistemológico } \\
\text { - Impacto } \\
\text { organizacional } \\
\text { - Impacto } \\
\text { ambiental } \\
\text { - Impacto social }\end{array}$ & $\begin{array}{l}\text { Internos } \\
\text { Alumnos, personal } \\
\text { docente/investigador, personal no } \\
\text { docente. } \\
\text { Externos } \\
\text { Egresados, empleadores, } \\
\text { competidores, competidores } \\
\text { locales, estado, }\end{array}$ \\
\hline
\end{tabular}




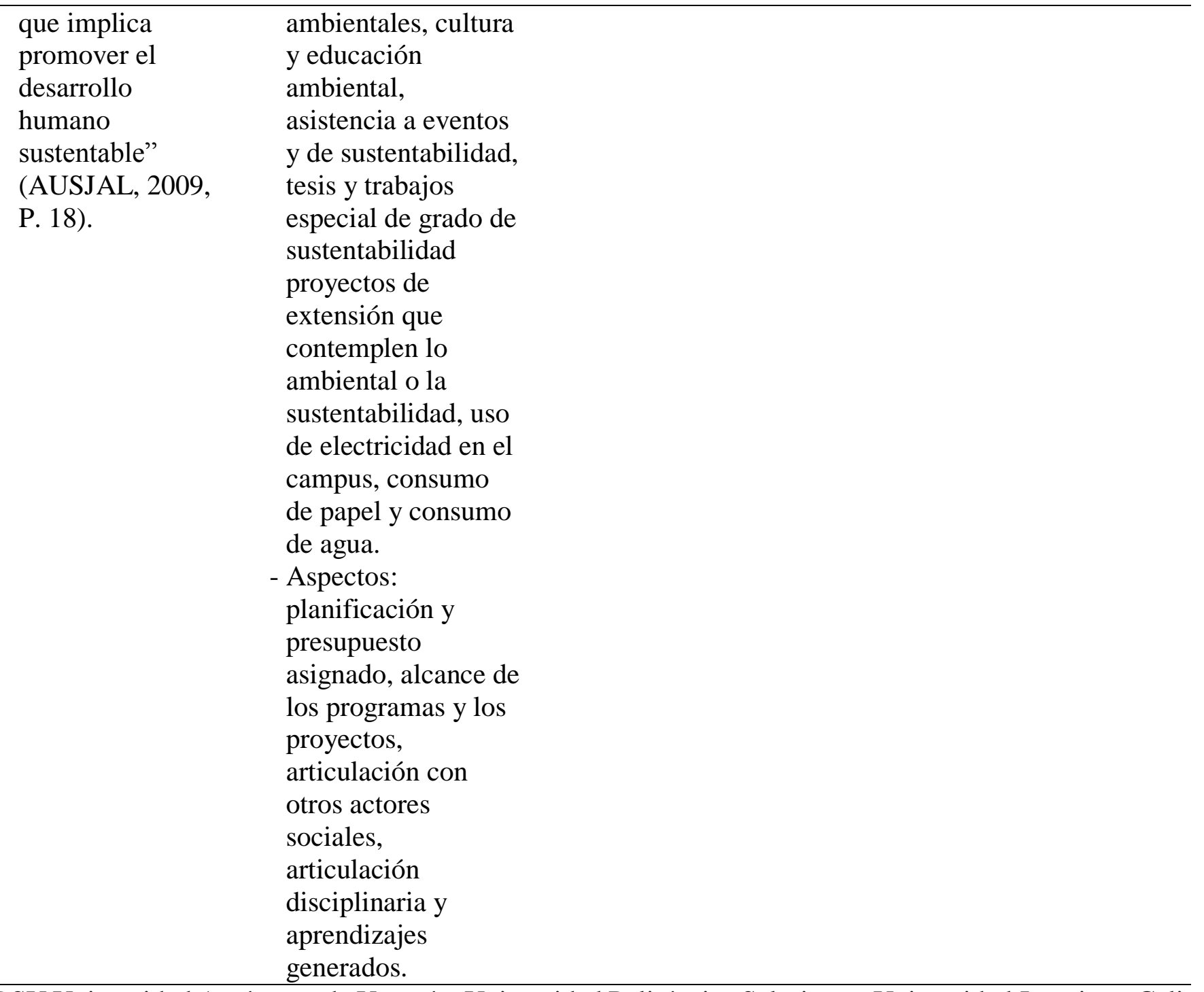

Fuente: modelo institucional de RSU Universidad Autónoma de Yucatán, Universidad Politécnica Salesiana y Universidad Javeriana-Cali. 
Con estos planteamientos se puede inferir que las IES están llamadas a ser promotoras de cambio en una sociedad que enfrenta desafíos estructurales importantes como el desempleo, la desigualdad, pobreza, crisis económicas, conflictos geopolíticos y un grave deterioro ambiental, por mencionar algunos, desde un marco de transparencia, ética, sustentabilidad, congruencia y credibilidad, al tiempo que los estudiantes sean forados bajo tales premisas (Misas, 2004).

Por su parte, la Junta Central de Contadores (JCC) resalta la importancia de que los contadores estén capacitados en administrar y asesorar negocios desde su estructura financiera para participar de manera activa en las campañas de Responsabilidad Social en empresas, especialmente, en las organizaciones del sector público donde las problemáticas por ausencia de la misma y la ética profesional han conllevado a que el desempeño de la profesión se vea comprometida en situación de riesgo, corrupción y trasgresiones al código de ética (Universidad Libre, 2018).

Al mismo tiempo, de acuerdo con Meseguer et al (2020), con relación a los Objetivos de Desarrollo Sostenible (ODS) para 2030, la educación permite la autosuficiencia, fomenta el crecimiento económico mejorando las habilidades y la vida de las personas. Así, la RSU y los grupos de interés requieren un mayor acceso la universidad, eliminando, entre otras, las desigualdades de género, la inseguridad alimentaria y los conflictos armados.

Por tanto, se observa que, desde su origen, las universidades han sido la base de la educación y, en cierta medida, de la sostenibilidad. En este contexto, las universidades están obligadas a implementar una serie de principios y valores éticos en los campos de la gestión, docencia e investigación en concordancia con su misión y visión institucional.

Así, en la sociedad del conocimiento, la RSU se refiere a la gestión del modelo estratégico en el contexto de una IES socialmente responsable. Por ello, la implementación de la RSU conlleva la autocrítica de la propia institución, evaluando responsablemente el impacto de sus acciones en la sociedad (Meseguer et al., 2020). 


\section{Metodología}

El documento se deriva de un proyecto de investigación profesoral, en el marco de cooperación académica entre programas de Contaduría Pública de la Universidad Mariana (Colombia) y Universidad César Vallejo (Perú).

La metodología se fundamenta en el paradigma cualitativo, de tipo descriptivo y con técnica de revisión documental, al realizar un acercamiento al estado del arte que permitió estructurar dimensiones y componentes de RSU y vincularlos con los dos programas de contaduría. Los estudios descriptivos buscan especificar las peculiaridades importantes de personas, grupos, comunidades o cualquier otro fenómeno que sea sometido al análisis (Dankhe, 1976).

La estructuración de dimensiones de la RSU se realizó a través de una revisión sistemática de la literatura a partir de artículos, libros y otros documentos publicados en bases de datos y páginas web. La búsqueda del término "responsabilidad social universitaria" y "responsabilidad social universitaria y contaduría pública" arrojó resultados que pertenecen a iniciativas y dimensiones específicas de responsabilidad social en instituciones de educación superior. Los textos se revisaron para filtrar la literatura relevante relacionada con la responsabilidad social universitaria en diferentes contextos aplicables a las universidades objeto de estudio.

Conjuntamente, con la revisión de la literatura se construye una matriz que incluye dimensiones y componentes de la RSU para en una futura investigación realizar un trabajo de campo en la escuela/programa de contaduría de las dos universidades analizadas y aplicar a sus comunidades universitarias (directivos, docentes y estudiantes).

\section{Resultados}

Una vez abordado el marco teórico y la metodología del artículo y para dar respuesta al objetivo que busca analizar los fundamentos teóricos sobre la RSU y vincularlos con programas de contaduría pública de las universidades estudiadas, se debe entrar a comprender que el mundo contemporáneo está sumido en un capitalismo exuberante, la ambición por el poder político y el domino económico ha permeado las distintas esferas de la sociedad, y en esta ruta, la profesión 
contable se ve inmersa en disyuntivas desestabilizadoras que conllevan a transgredir los códigos de ética cuando los profesionales operan por encima de sus valores, situación que se presenta en diversas naciones de Latinoamérica, soslayando el interés público y en detrimento tanto de la profesión contable como de los entes económicos que se ven afectados por la manipulación de los estados financieros (Corporación Latinobarómetro, 2018; Fernández y Campo, 2017; Pinilla y Álvarez, 2013).

De lo anterior surge la importancia que las universidades conjuguen adecuadamente sus funciones sustantivas (docencia, investigación y extensión) con la política transversal de RSU; así las universidades latinoamericanas tienen como finalidad impartir educación superior para formar personas, para una sociedad que enfrenta enormes desafíos: pobreza, desigualdad, violencia y la vida misma en el planeta (Chavarría \& Arroyo, 2018).

Si el reto es formar profesionales de la ciencia contable con sólidos conocimientos en la gestión financiera, el aseguramiento y asesoría (tributaria, contable, en control interno, informes no financieros, entre otros) unido a la formación humanista con un verdadero compromiso con la sociedad; la universidad esta llamada a ser ejemplo de responsabilidad, factor de una buena educación y clave para transformar el mundo.

Parafraseando a Vallaeys y Álvarez (2019), la responsabilidad social, es un compromiso de todos, inherente a la función formativa de la universidad y transversal en sus dimensiones sustanciales (docencia, investigación y extensión), para contribuir al desarrollo sostenible y considerando las expectativas de las partes interesadas. En este orden de ideas, y con el fin de responder a las apremiantes necesidades en lo económico, social y ambiental, la universidad del siglo XXI, debe impulsar cambios en su gestión administrativa, el diálogo permanente con grupos de interés, revisión de su modelo pedagógico y mejores relaciones con la sociedad al definir políticas de RSU vinculadas con su contexto y los retos del mundo actual, desde dimensiones económicas, sociales y ambientales.

Lo anterior se integra a la función sustantiva de extensión, considerada como la "apertura de datos en el nivel de la educación superior como una estrategia que facilita la relación de la comunidad 
universitaria con el entorno, así como su participación activa en la búsqueda de soluciones a problemas internos y externos" (Osorio et al., 2020, p. 275).

Avanzando en el tema, la RSU se constituye en eje articulador de la gestión misional de las instituciones de educación superior, situación que confiere un papel primordial en la definición del quehacer universitario. De acuerdo con Gaete (2015) "el concepto de RSU se encuentra relacionado con la permanente preocupación por los impactos del quehacer universitario en la sociedad" (p. 98); así las cosas, se debe formar un ser social que identifique los problemas del medio en el que vive, como deterioro del ambiente, problemas de pobreza, corrupción, violación de derechos humanos, inequidad de género, deterioro ambiental, entre otros. Por tanto, este documento, presenta como primer resultado, realizar un acercamiento a la literatura y reconocer la importancia de incorporar prácticas y estrategias de RSU en las universidades y específicamente, en la formación del contador público como garante de fe pública y promotor del interés público.

En la actualidad la RSU se ha convertido en un concepto cada vez más importante en Latinoamérica y en resto del mundo, ha sido y será parte del debate sobre calidad educativa, competitividad y sostenibilidad en el contexto de la globalización, conjuntamente, refleja la necesidad de defender los valores comunes y aumentar el sentido de solidaridad y cohesión social. Así mismo, la RSU implica que las universidades deban comportarse de manera responsable donde sea que operen y con base en las normas y estándares que apliquen a nivel nacional e internacional (Meseguer et al., 2020).

Sin embargo, al apostar por una mejor interacción de la universidad como centro de formación, pensamiento y generación del conocimiento, es necesario avanzar desde el modelo pedagógico y las prácticas de responsabilidad social en formas alternativas que promuevan el pensamiento crítico, con fundamento en la ética y en contra de modelos como el desarrollismo, para reflexionar en torno a estructuras dominantes de la matriz capitalista y su fase neoliberal.

Lo anterior lleva a resignificar la proyección social y estructuras educativas conservadoras, al respecto Alvarado et al. (2017) afirman: "La discusión teórica sobre la RSU en las instituciones 
de educación superior (IES) es un tema de diversos debates teóricos y metodológicos, los enfoques que argumentar las formas de medir su calidad y pertinencia en un sistema educativo" (p. 42).

En este sentido, se requiere avanzar en la preservación y valoración de la identidad, una educación para la interculturalidad, estudio y análisis del contexto y los conflictos sociales de las últimas décadas, en definitiva, la construcción de nuevos saberes y métodos para trabajar con los grupos de interés que permitan priorizar necesidades y fomentar sinergias interinstitucionales (universidad, empresa, estado y comunidad), formando ciudadanos responsables y comprometidos con el desarrollo sostenible.

Conjuntamente, la exploración de textos permitió estructurar una matriz con las principales dimensiones, subdimensiones y componentes de la RSU, tal como se evidencia en la Tabla 3, que permitirán analizar el grado de avance sobre las prácticas de RSU en la escuela/programa de las dos universidades analizadas, así:

\section{Tabla 3}

Dimensiones de la Responsabilidad Social Universitaria

\begin{tabular}{|c|c|c|c|c|}
\hline \multicolumn{5}{|c|}{ Dimensiones de la responsabilidad social universitaria (RSU) } \\
\hline Subdimensiones & $\begin{array}{c}\text { Funcionamiento } \\
\text { organizacional }\end{array}$ & Educativa & Extensión & Investigación \\
\hline \multirow{8}{*}{ Componentes } & $\begin{array}{l}\text { Adopción } \\
\text { institucional }\end{array}$ & $\begin{array}{l}\text { Estructura } \\
\text { pedagógica }\end{array}$ & $\begin{array}{l}\text { Accesibilidad } \\
\text { social a la } \\
\text { universidad }\end{array}$ & $\begin{array}{l}\text { Generación } \\
\text { social del } \\
\text { conocimiento }\end{array}$ \\
\hline & $\begin{array}{l}\text { Contratación de } \\
\text { personal }\end{array}$ & $\begin{array}{l}\text { Competencias } \\
\text { de estudiantes }\end{array}$ & & \\
\hline & & Docencia & & \\
\hline & Ética & $\begin{array}{l}\text { Educación } \\
\text { contable }\end{array}$ & & \\
\hline & $\begin{array}{l}\text { Ambiente } \\
\text { organizacional }\end{array}$ & $\begin{array}{l}\text { Formación para } \\
\text { la fe pública }\end{array}$ & & \\
\hline & Talento humano & & & \\
\hline & $\begin{array}{l}\text { Gestión de la } \\
\text { Comunicación }\end{array}$ & & & \\
\hline & $\begin{array}{l}\text { Bienestar a } \\
\text { estudiantes y relación } \\
\text { grupos de interés }\end{array}$ & & & \\
\hline
\end{tabular}

Fuente: elaboración propia con datos de Vallaeys, De la Cruz, \& Sasia (2009). 
Estas dimensiones, en su conjunto, permiten considerar las funciones sustantivas de la universidad, teniendo en cuenta que la RSU debe ser transversal a las actividades que realizan las IES, siendo las variables: funcionamiento organizacional, educativa, extensión y organización; estas a su vez se dividen en 16 componentes: adopción institucional, contratación de personal, equidad remunerativa, principios éticos, ambiente laboral, oportunidades laborales, capacitación del personal, comunicación interna y grupos de interés, bienestar a estudiantes, estructura pedagógica, competencias de estudiantes, docencia, educación contable, formación para la fe pública, accesibilidad social a la universidad y generación social del conocimiento.

Esta matriz a diferencia de las planteadas por diversos autores, reporta dimensiones y componentes que nutren cada ítem para una mayor comprensión de las actividades que realizan las universidades en materia de RSU.

Por su parte, la medición de la RSU es un asunto complejo, el concepto por ser extenso y transversal a diversas funciones en las universidades, requiere de instrumentos que permitan una evaluación adecuada para las actividades de RSU (Villarreal, 2014). Como primera medida es necesario un instrumento con enfoque cualitativo para obtener información de gestión en las IES. Estos se obtienen de los informes de gestión proporcionados en la página web de la institución. Adicional, requiere de una medición cuantitativa con el ánimo de obtener datos estadísticos sobre el nivel de exposición que tienen los grupos de interés a las actividades de RSU realizadas por las IES, al respecto la Tabla 3 planteada permitirá diseñar los instrumentos para el trabajo de campo. Por último, con las dimensiones anteriormente expuestas se diseñará un instrumento que permita medir la percepción de docentes, estudiantes y administrativos sobre la RSU en los programas de contaduría pública en la Universidad Mariana (Colombia) y Universidad César Vallejo (Perú) según los planteamientos de Baca et al. (2017).

Al mismo tiempo, vincular la RSU con la profesión contable permite fortalecer la confianza pública (del actuar de la universidad en la sociedad, medio ambiente y gestión empresarial), el saber y quehacer contable para que los contadores estén capacitados en administrar, gestionar y asesorar negocios más allá del modelo financiero internacional, responsabilidades tributarias, 
diseño y evaluación de controles y decisiones financieras para motivar la participación activa en campañas de responsabilidad social en las empresas (Osorio \& Poveda, 2016).

Así, el estudiante de contaduría, desarrollará buenas prácticas de RSU para generar verdaderas transformaciones y avanzar hacia el desarrollo sostenible, entonces los futuros profesionales requieren ser formados en IES que asuman la RSU como un eje transversal en sus funciones sustantivas y su quehacer como universidad, de tal forma que su perfil de egreso corresponda a los requerimientos del mundo contemporáneo y se conviertan en actores garantes de cambio dentro de las organizaciones del sector público, privado y la (re) construcción de una sociedad más solidaria y justa.

La tarea a emprender requiere cambios sustanciales en la manera de educar, desarrollar competencias y aprendizajes desde el aula (presencial o virtual), junto a la investigación y proyección social con políticas y acciones de RSU, que permita fortalecer el perfil profesional, donde la promoción del interés público, ejercicio ético y calidad del trabajo.

En consecuencia, formar integralmente frente a las responsabilidades sociales, compromiso con la paz, respeto a la diversidad, calidez humana al tratar con empatía a los demás y el interés por un verdadero progreso sin dejar a nadie atrás, se integra con el verdadero sentido de la RSU a partir de un buen gobierno corporativo universitario con transparencia y autonomía de su gestión educativa para fortalecer la confianza que la comunidad tiene en estas instituciones, respondiendo a las nuevas demandas sociales, a partir de políticas de calidad e interacción con los grupos de interés, que permita una visión compartida y mayor participación en la toma de decisiones donde se alcance efectividad y legitimidad.

En definitiva, la educación contable y la RSU están enfocadas al estudio y comprensión de las problemáticas y oportunidades del contexto, la realidad empresarial y socio ambiental, unido al fomento del liderazgo, apropiación de la tecnología y pertinencia para un mejor desempeño del profesional contable con visión nacional e internacional frente a un mundo cambiante, complejo y de incertidumbres. 


\section{Conclusiones}

Una vez analizadas las dimensiones de la RSU se relacionan con la formación del contador público, atendiendo las necesidades del contexto socio-empresarial; esto conlleva a una reflexión sobre la calidad de la educación contable, representada en aspectos como el currículo, los contenidos de las asignaturas para el fomento de las prácticas de responsabilidad, la promoción de un pensamiento integrador para el desarrollo sostenible y el fortalecimiento del interés público.

De esta manera el futuro contador, tendrá mejores competencias ciudadanas y habilidades para el trabajo interdisciplinario. Como plantea Gaete (2015), la RSU vinculada con el perfil profesional y formación del talante ético, debe ir más allá de estándares educativos tradicionales, para analizar con la comunidad, sus oportunidades y dificultades, de forma proactiva frente a la búsqueda de soluciones concertadas que permita cerrar brechas, vinculado con una actitud ética y del servicio, para el cuidado de sí mismo, de sus semejantes y la naturaleza (flora y fauna).

Lo anterior le permitirá en su desempeño profesional comprender integralmente a la entidad y su contexto, estar preparado para enfrentar dificultades, asumir retos, buscar, analizar y divulgar información contable, tributaria, financiera y no financiera, la promoción de un control estratégico y análisis de riesgos; que en su conjunto permita fortalecer la confianza y mejor toma de decisiones. A lo anteriormente señalado, se coligen los desafíos en la educación contable desde el nivel superior, que al decir de Jarrín (2019) son: economía (creación de riqueza para todos, mediante medios de consumo y de producción sostenibles); Equidad (participación de todos los grupos sociales); y Medio ambiente (conservación y gestión de los recursos). (p.20).

En síntesis, las IES debe enfocar sus estrategias a la formación de estudiantes de manera holística en tres categorías: aprendizaje para el servicio, fortalecimiento de la ciudadanía y rol de los profesionales (Gaete, 2015). De acuerdo con Tobón et al. (2019) esta formación está relacionada con el desarrollo de interpretaciones de las problemáticas sociales de las comunidades, su comprensión aunada a la transferencia de conocimiento, hacia la promoción de la innovación social, resulta fundamental para el desarrollo y el logro de la RSU. 
Conjuntamente, la dimensión de responsabilidad social en las instituciones de educación superior está siendo llamada a la vanguardia del cambio, tanto en el sentido de repensar sus prácticas internas como su interacción con la comunidad circundante (mediante el establecimiento de asociaciones más sólidas).

Las actividades y proyectos de RSU se consideran motores de cambio para las universidades, sin embargo, aún no está suficientemente estudiado el potencial que puede tener la percepción de la comunidad universitaria en esta área, en sus capacidades académicas y profesionales (Coelho \& Menezes, 2020). Por tanto, se hace necesario investigar y generar proyectos en alianzas con otras IES en las escuelas/programas de contaduría, para analizar el impacto de las prácticas de RSU sobre las funciones sustantivas y generar estrategias de respuesta ante las nuevas exigencias que suscita el mundo contemporáneo.

En este orden de ideas, la vinculación de la RSU en la formación del contador permitirá a los grupos de interés internos (estudiantes, directivos y docentes) y externos (comunidad, gobierno, empresas públicas y privadas) contar con IES mejor administradas, con un mayor compromiso social, promoción de valores identitarios del contexto donde interactúan y el mejoramiento continuo de las relaciones con la sociedad, para avanzar en la transformación social y creación de valor compartido.

En suma, la multiplicidad de conceptos sobre RSU hacen indispensable que los planteamientos teóricos se clasifiquen para una mejor comprensión desde un enfoque organizacional (tradicional) y/o de transformación (enfoque crítico) y de los impactos que se pueden generar en la estructura organizacional y gobernanza universitaria, desarrollo humano de su personal, la formación de estudiantes; el enfoque investigativo para llegar a un consenso general que permita la aplicación en las funciones sustantivas de las IES. Desde esta óptica, generar respuestas a las verdaderas necesidades desde lo social, ambiental y económico, así como las alianzas que surgen de la interacción con el entorno social, redes y participaciones sociales, culturales y políticas.

Por tanto, el presente escrito como aporte, identificó dimensiones, subdimensiones y componentes de la RSU basados en el desarrollo conceptual y teórico para ser integrados como ejes articuladores 
en los modelos de gestión de las escuelas/programas de contaduría objeto de estudio, esto con el fin de gestionar procesos de formación profesional en los estudiantes y desempeño de las actividades de docentes y directivos, concientizándolos del rol que ejercen como ciudadanos del mundo y como tal de los impactos que generan con su actuar cotidiano.

Lo anterior, analizado desde el proceso de formación contable para la gestión universitaria, requiere el compromiso de toda la comunidad universitaria, considerando la importancia de su ejecución, desde la gestión directiva para vincularlo con el perfil profesional y el servicio, la solidaridad que impulse transformaciones, el fortalecimiento de la ciudadanía y un mejor desempeño de los profesionales (Gaete, 2015).

Finalmente, es necesario que los futuros Contadores Públicos sean formados en IES que incluyan prácticas de RSU, para una mejor interacción con el entorno institucional y socio empresarial, de tal forma que su perfil de egreso corresponda a los requerimientos del mundo contemporáneo y se conviertan en actores garantes de transformaciones sociales y cuidado ambiental.

\section{Referencias}

(1) Agudelo, M. \& Viloria, N. (2017). Las virtudes de la práctica contable. Un análisis desde MacIntyre. Revista Argentina de Investigación en Negocios, 3(1), 59-72. http://ppct.caicyt.gov.ar/index.php/rain/article/view/V3N1-2a06/10175

(2) Alvarado, E., Morales, D. \& Ortiz, J. (2017). Un análisis sobre la percepción que los directivos y docentes tienen de la responsabilidad social universitaria en las facultades de contaduría y administración en México. Universidad \& Empresa, 19(32), 37-59.

(3) Amato, C. N., Buraschi, M. \& Peretti, M. F. (2016). Orientación de los empresarios de Córdoba-Argentina hacia la sustentabilidad y la responsabilidad social empresarial: identificación de variables asociadas a cada constructo. Contaduría y Administración, 61, 84105.

(4) Aristimuño, M. \& Rodriguez, C. (2014). Responsabilidad social universitaria. Su gestión desde la perspectiva de directivos y docentes. Estudio de caso: una pequeña universidad latinoamericana. Interciencia, 39(6), 375-382. 
(5) ASCUN. (2007). Asociación de Universidades de Colombia. Obtenido de www.ascun.org.co

(6) AUSJAL. (2007). Asociación de Universidades Jesuitas de America Latina. www.ausjal.org/

(7) Baca, H., Rondán, F. \& García, J. (2017). Propuesta de medición de la responsabilidad social universitaria. Revista Espacios, 38(43), 12-49.

(8) Bowen, H. (1953). Social Responsibilities of the businessman. Nueva York: Harper.

(9) Brijaldo, M. \& Pérez, E. (1 de enero de 2017). La ética y responsabilidad social del contador público como aporte a la construcción de una mejor sociedad. Universidad de la Salle. https://ciencia.lasalle.edu.co/cgi/viewcontent.cgi?article=1700\&context=contaduria_publica

(10)Calle, D. \& Santacruz, T. (2011). Modelo de Responsabilidad Social Universitaria aplicado en la Universidad Politécnica Salesiana sede Cuenca. https://dspace.ups.edu.ec/bitstream/123456789/1331/11/UPS-CT002239.pdf

(11)Chavarría, L. \& Arroyo, C. (2018). Educar para el emprendimiento social, un reto para la universidad latinoamericana: macroproyecto de investigación de la Alafec. Ciudad de México: Publicaciones Empresariales UNAM, FCA Publishing. ISBN: 6073012055

(12) Coelho, M. \& Menezes, I. (2020). University social responsibility as a driving force of change: students' perceptions beyond the ivory tower. On the Horizon, 28(2), 93-100. doi: https://doi.org/10.1108/OTH-02-2020-0005

(13) Congreso de la República. (13 de diciembre de 1990). Ley reglamentaria de la profesión de Contador Público. Ley 43 de 1990.

(14)Congreso de la República del Perú. (11 de Septiembre de 1959). Ley No. 13253. Obtenido de Ley de Profesionalización de los Contadores: https://www.ccplamb.org.pe/admin/document/documentos/archdoc_ccpl_01.pdf

(15) Congreso de la República del Perú. (16 de enero de 2007). Ley 28951. Obtenido de Ley de actualización de la Ley No. 13253 de profesionalización del Contador Público y de creación de los colegios de contadores públicos: http://www.oas.org/juridico/spanish/mesicic3_per_ley28951.pdf

(16) Corporación Latinobarómetro. (2018). Informe 2018. Santiago de Chile: Latinobarómetro. https://www.latinobarometro.org/latNewsShowMore.jsp?evYEAR=2018\&evMONTH=-1

(17) Dankhe, G. (1976). Diferentes diseños - tipos de investigación . México, DF: McGraw Hill.

(18)De Sousa, B. (2010). La universidad en el siglo XXI. Para una reforma democrática y emancipadora de la universidad. Uruguay: Coedición Extensión universitaria. Universidad 
de la República - Ediciones Trilce.

(19)Escorcia, R., Gutiérrez, A. \& Henriquez, H. (2007). La educación superior frente a las tendencias sociales del contexto. Educación y Educadores, 10(1), 63-77. http://www.scielo.org.co/pdf/eded/v10n1/v10n1a06.pdf

(20) Fernández, I. \& Campo, J. (2017). Dilemas del contador y quebrantamiento al código ético entre los años 2012-2014. Gestión y desarrollo, 13, pp. 43-53. ISSN: 0123-5834

(21) Foster, H. (2002). Introducción a la posmodernidad. Editorial Universidad Internacional de la Rioja, La posmodernidad (pp. 7-18). ISBN: 84-7245-154-2

(22) Gaete, R. (2011). Identificación de los stakeholders de las universidades. Revista de Ciencias Sociales, 17(3), 486-499.

(23) Gaete, R. (2015). El concepto de responsabilidad social universitaria desde la perspectiva de la alta dirección. cuadernos de administración, 31(53), 97-107.

(24) García, J. \& Zabala, H. (2008). Políticas de Estado como sustento de la organización ciudadana. Revista del Centro de Investigación de Ciencias Administrativas y Gerenciales, 1(5), 113-131. https://dialnet.unirioja.es/servlet/articulo?codigo=3216837

(25) Gasca, O. (2011). Construir ciudadanía desde las universidades, responsabilidad social universitaria y desafíos ante el siglo XXI. Convergencia, 18(56), 37-58.

(26) Hill, R. (2004). The socially responsible university: Talking the talk while walking the walk in the college of business. Journal of Academic Ethics, 2(1), 89-100.

(27) International Accounting Education Standards Board (2015). Consejo de Normas Internacionales de Educación Contable. https://www.iaesb.org/news-events/2014-01/iaesbpublica-nuevos-est-ndares-sobre-el-contenido-del-programa-de-educaci

(28) Jarrín, F. (2019). La responsabilidad social universitaria en la formación del contador. En P. U. Ecuador, Las tendencias en Contabilidad y Gestión Empresarial. Visión de Iberoamérica (pp. 18-25). Quito: Centro de publicaciones Pontificia Universidad Católica del Ecuador.

(29) Junta de decanos de los colegios de contadores públicos. (2005). Resolución No. 013-2005JDCCPP. Obtenido de Código de ética profesional del contador público peruano: http://jdccpp.org.pe/docs-publicaciones/codigo_etica_contador_publico_peruano.pdf

(30) Lanz, R. (2003). La universidad se reforma. Michigan, EEUU: Universidad Central.

(31)Leff, E. (2013). SABER AMBIENTAL: Sustentabilidad, racionalidad, complejidad, poder. México: Universidad Autónoma de México. 
(32)Londoño, J. \& Bermúdez, L. (2016). Análisis del concepto de interés público a partir de la discusión entre los presupuestos planteados por las corrientes funcionalista y crítica de la contabilidad. Revista Contaduría, 1(69), 13-31.

(33)López, O. (2013). Responsabilidad social en el ejercicio de la revisoría fiscal. Contaduría Universidad de Antiquia, 107-124.

(34) Medina, R., Franco, M., Torres, L., Velázquez, K. \& Valencia, M. (2017). La responsabilidad social universitaria en la actual sociedad del conocimiento. Un acercamiento necesario. Medisur, 15(6), 786-791.

(35) Meseguer, V., Abad, E., Belmonte, L. \& Molina, V. (2020). Examining the Research Evolution on the Socio-Economic and Environmental Dimensions on University Social Responsibility. International Journal of Environmental Research and Public Health, 17(4229), 1-30. doi:10.3390/ijerph17134729

(36) Misas, G. (2004). La educación superior en Colombia. Análisis y estrategias para su desarrollo. Bogotá: Universidad Nacional de Colombia. ISBN: 958-701-356-5

(37) Muhammad, A., Ishamuddin, M., Sharina, O. \& Umar, H. (2021). University social responsibility: A review of conceptual evolution and its thematic analysis. Journal of Cleaner Production, 286(1). doi: https://doi.org/10.1016/j.jclepro.2020.124931

(38) Navarro, J. \& Saavedra, Y. (2016). La importancia de la responsabilidad social del Contador Público en su quehacer profesional. [Tesis de pregrado, Fundación Universitaria los Libertadores] Bogotá, Colombia: Fundación Universitaria los Libertadores.

(39) Osorio, A. \& Poveda, A. (2016). Importancia de la ética del Contador Público frente a la Responsabilidad Social Empresarial en Colombia. Bogotá: Universidad Libre.

(40) Osorio, M., Amaya, F. \& González, M. (2020). Análisis de datos abiertos de instituciones de educación superior colombianas como apoyo a la relación Universidad-Entorno. Entramados, 16(1), 272-284.

(41) Pinilla, J. \& Álvarez, J. (2013). Del Contador Público y la ética profesional: un dilema inmerso en un ambiente saturado de conflictos morales. Contaduría Universidad de Antioquia (63), $127-158$.

(42) Piña, I. (2006). La universidad en el siglo XXI. Revista de educación superior, 35(138), 115122. ISSN: 0185-2760

(43) Pontificia Universidad Javeriana. (2014). Estudio sobre la Responsabilidad Social 
Universitaria en la Pontificia Universidad Javeriana. Oficina para el fomento de Responsbilidad Social

Universitaria. https://www.javeriana.edu.co/documents/15838/7332942/Estudio+sobre+RSU+en+la+PUJ \%2C\%202013-2014.+Comunidad+Educativa/200f6f8f-05bf-43d4-a327-7d106f4bf8e9

(44) Pulgarin, F. \& Zapata, L. (2014). Incidencia del ejercicio profesional del contador público en la continuidad de las MIPYMES en Colombia. Contaduría Universidad de Antioquia, 1(64), pp. 181-206.

(45) Quintero, J. (30 de Agosto de 2018). Responsabilidad social del contador según la IFAC. Revista de estudiantes de contaduría pública, (20), 1-5. https://revistas.udea.edu.co/index.php/adversia/article/view/334470

(46) Teran, A. \& Torres, A. (2020). University Social Responsibility (USR) and Its Mission: The case of the Universidad Panamericana in Mexico. En A. García, \& C. Atristain, Strategy, Power and CSR: Practices and challenges in Organizational Management (235-257). Emerald Publishing Limited.

(47) Tobón, F., López, L. \& Londoño, C. (2019). Investigación formativa y prácticas académicas integradoras en el marco de la Responsabilidad Social Universitaria: Un análisis a partir de metodología mixta. Entramados, 15(2), 188-200.

(48) UNESCO. (11 de noviembre de 1998). Conferencia mundial sobre educación superior en el siglo XXI visión $\quad y \quad$ acción. http://www.unesco.org/education/educprog/wche/declaration_spa.htm.

(49) Universidad Autónoma de Yucatan. (2014). Plan de Desarrollo Institucional 2014-2022. Acción 10: Modelo de Responsabilidad Social Universitaria. Yucatan: Universidad Autónoma de Yucatan.

(50) Universidad Construye País. (2006). Hacia las sociedades del conocimiento. Obtenido de Informe Mundial. Observando la Responsabilidad Social Universitaria. Santiago de Chile: http://rsuniversitaria.org/web/images/sto-ries/memoria/UCP\%202006.pdf

(51) Universidad Libre. (27 de febrero de 2018). Informe revela las cifras del panorama actual de los Contadores Públicos en Colombia. http://www.unilibre.edu.co/la-universidad/ul/998informe-revela-las-cifras-del-panorama-actual-de-los-contadores-publicos-en-colombia

(52) Vallaeys, F. (2014). La responsabilidad social universitaria: un nuevo modelo universitario contra la mercantilización. Revista Iberoamericana de Educación Superior (RIES), 5(12), 
105-117. http://ries.universia.net/index.php/ries/article/view/439

(53) Vallaeys, F. \& Álvarez, J. (2019). Hacia una definición latinoamericana de Responsabilidad Social Universitaria. Aproximación a las preferencias conceptuales de los universitarios. Educación XXI, 22(1), 93-116. doi: 10.5944/educXX1.19442

(54) Vallaeys, F. \& Carrizo, L. (2006). Responsabilidad Social Universitaria: marco conceptual, antecedentes, herramientas. Lima, Perú: Banco Interamericano de Desarrollo.

(55) Vallaeys, F., De la Cruz, C. \& Sasia, P. M. (2009). Responsabilidad Social Universitaria. Manual de primeros pasos. México: McGraw.

(56) Vasilescu, R., Barna, C., Epure, M. \& Baicu, C. (2010). Developing university social responsibility: A model for the challenges of the new civil society. Procedia Social and behavioral sciences, 2, 4177-4182. doi: 10.1016/j.sbspro.2010.03.660

(57) Vélez, G. \& Ruiz, G. (2019). La universidad en crisis, ¿amenaza o reafirmación de su ontología? Revista de la educación superior, 48(190), 1-22. ISSN: 0185-2760

(58) Villarreal, A. (2014). Avanzando en el tema, la medición de la RSU es un asunto complejo, el concepto por ser extenso y transversal a diversas funciones en las universidades requiere de instrumentos que permitan una evaluación adecuada para las actividades de RSU. [Tesis doctoral, Universidad Internacional de Cataluña]. Barcelona, España: Universidad Internacional de Cataluña. https://www.tdx.cat/bitstream/handle/10803/283417/Tesi_Azul_Alf\%C3\%A9rez_Villareal.p df? sequence $=1 \&$ is Allowed $=\mathrm{y}$.

(59) Wolff, L. F. (2009). Reflexiones sobre la propuesta de Boaventura de Sousa Santos para la universidad del siglo XXI. Uni-pluri/versidad, 9(2), 1-5.

(60)Zúñiga, L., Vázquez, E. \& Cuatepotzo, J. (2019). Instituto Méxicano de Contadores Públicos. Contaduría Pública. https://contaduriapublica.org.mx/2019/06/01/desafios-de-laresponsabilidad-social-universitaria-para-la-formacion-profesional-del-contador-publico

Cómo citar este artículo: Villarreal, J., Reyes, C. y Mucha, Á. (2021). Acercamiento teórico de la responsabilidad social universitaria (RSU) en programas de Contaduría Pública. Tendencias, 23(1), 341-371. https://doi.org/10.22267/rtend.222301.193 